Please send trade news information and illustrations to Arveen Bajaj at the $B D J$, Nature Publishing Group, The Macmillan Building, 4-6 Crinan Street, London N1 9XW.

Trade news is provided as a service to readers using text and images from the manufacturer, supplier or distributor and does not imply endorsement by the $B D J$. Normal and prudent research should be exercised before purchase or use of any product mentioned.

\section{Snappy restorations}

The Snappy Abutment from Nobel BiocareSnap makes it easy and simple to create beautiful aesthetics and worry-free impressions

It is suitable for posterior, partially edentulous and single implant restorations as the Snappy Abutment captures the margin in a pre-made impression coping.

The impression coping retracts the tissue, so no retraction cord is needed and the short profile means there is no need to make any modifications. Lastly, everything you need to place the abutment and record an impression, including the impression coping and healing cap is included in the package.

Reader response number 50

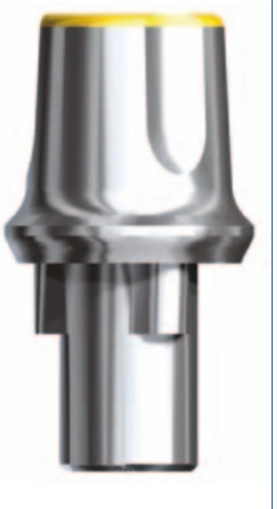

\section{Leading with linen}

Exclusive to Florence Roby is the very latest linen weave fabric. It has been designed into the brand new Pathos Tunic, exquisitely finished with an elegant diamante button. Florence Roby's new linen weave fabric is also easy care and despite its linen appearance, needs minimal ironing.

The classically cut Pathos Tunic is

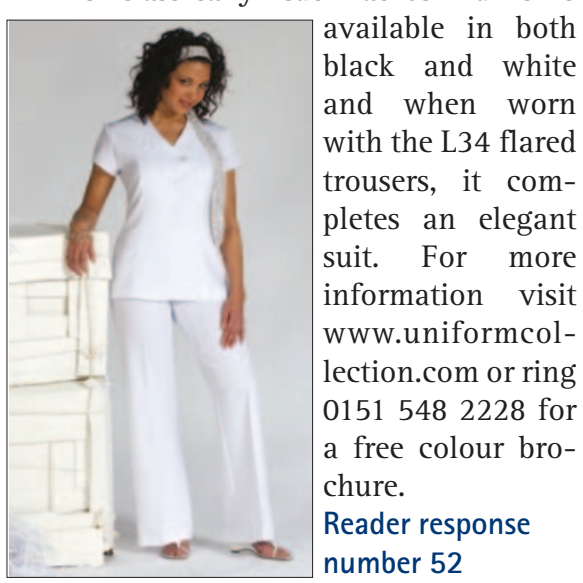

\section{TRADE NEWS}

WHAT'S NEW

\section{New ultra-bright dental loupe}

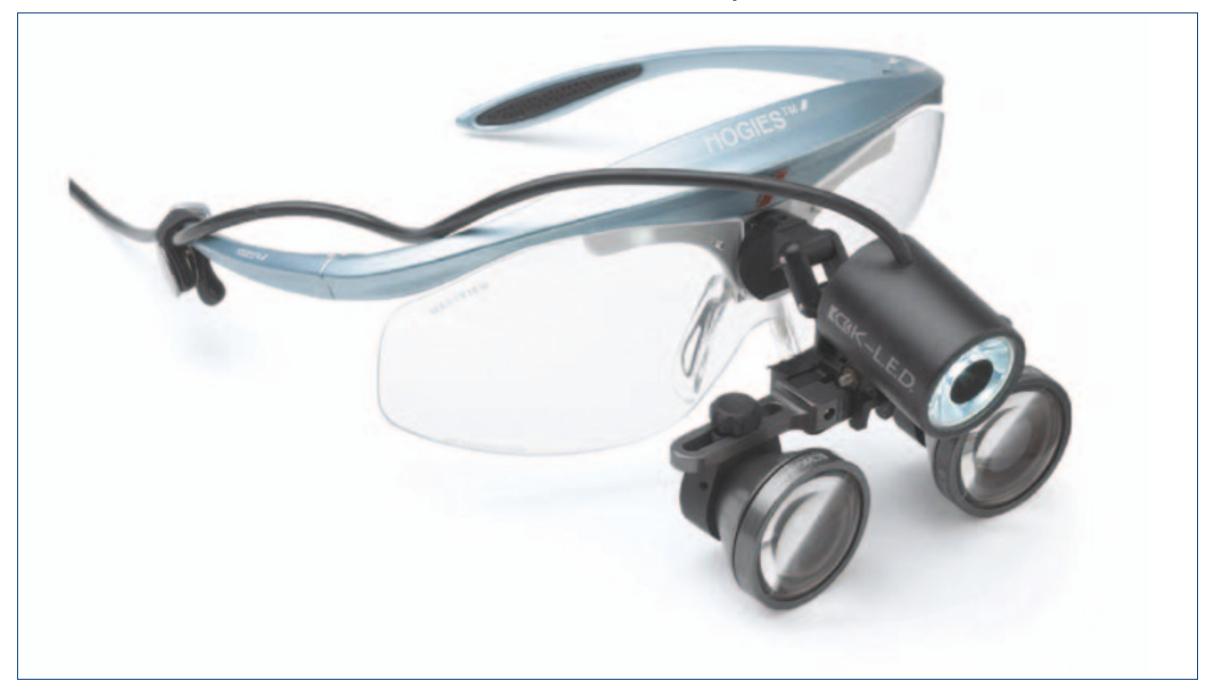

The new K-LED dental loupe light from Keeler provides an 18,000 lux patch of bright, homogenous light. The light is simply clipped to the front of the loupe bar and is powered by a small, rechargeable lithium battery which provides over four hours of continuous use.

Two batteries and a desk-top charging unit, which doubles as a holder for the batteries and loupes, are supplied as standard. This means one battery can be recharging while the other is in use. The K-LED is easy to fit and can be retrofitted to all Keeler loupe and frame combinations. An optional universal clip also allows it to be fitted to other manufacturers' loupes. Reader response number 51

\section{Cutting down on missed appointments}

A new text messaging service has been launched with the aim of reminding patients about their dental and medical appointments. The Messaging Centre (TMC) is a web-based text messaging platform designed to enhance business communications and continuity within Government healthcare departments, private GP Practices, dental surgeries and hospitals or anyone with a company or personal mobile phone.

The service has been designed specifically to benefit industries such as healthcare and pharmaceutical industries by providing a corporate text messaging service that can dramatically improve business operations, communications and provide enhanced aftercare for outpatients. The company was launched following research that found around 15 million medical appointments were missed each year costing the government and private healthcare industry millions in lost annual revenue.

The two-way text messages can be sent directly from many different platforms such as PCs, wireless PDAs (Personal Digital Assistant), company or personal GSMs (Global Systems for Mobile communications).

The Messaging Centre features include accessing the system from any internet connected PC, no set up fee and no minimum monthly spend, total control over who sends messages, there is no contract term and encryption ensures your company's data is kept confidential. For more information visit www.tmcsms.co.uk. Reader response number 53

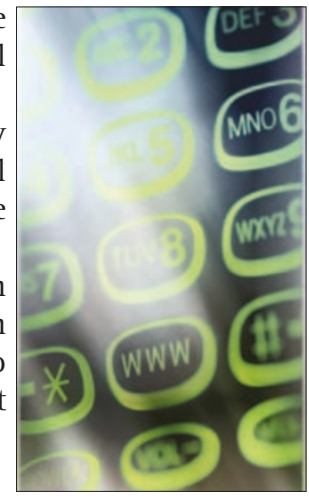




\section{Natural florescence}

NobelRondo Zirconia, from Nobel Biocare offers dental ceramists a professional, yet easy to use system to create beautiful restorations that mimic the natural florescence of real teeth. Made from zirconia, one of the world's strongest materials, the porcelain has a flexural strength of 120MPa. For use on Zirconia restorations, this unique porcelain includes six harmonic colour segments, logically arranged in an ergonomic tray. Combined with the unique layering technique, NobelRondo Zirconia provides the highest aesthetic results.

Reader response number 54

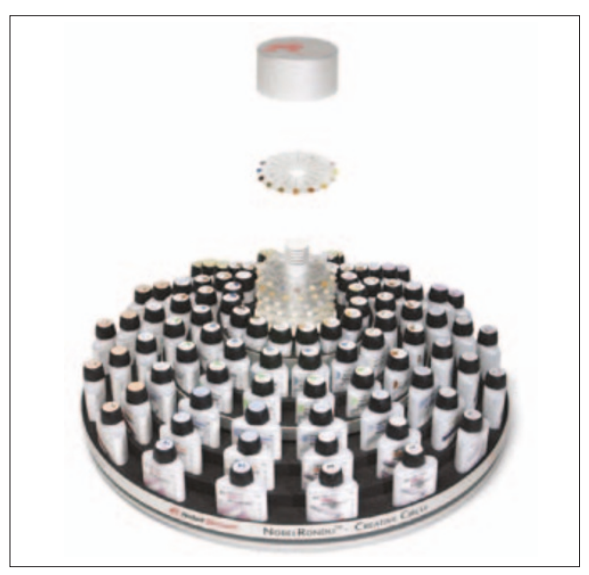

\section{Increased adhesion}

The tray adhesives Fix and Silfix from Dentsply ensure strong retention of impression material, be it alginates or A-silicones, inside the tray. Fix Adhesive (liquid and spray) are solvent-based adhesives which increase the adhesion between alginate impression materials and the impression tray surface. Fix Solvent is a spray to remove any remaining adhesive from the impression tray after impression taking and casting.

Silfix, the tray adhesive for A-Silicone impression materials, is a polysiloxane-based adhesive. Silfix is painted on the inner surface of the impression tray prior to application of the mixed impression material, in order to increase the adhesion to the tray of conventional, quadrafunctional and hydrophilic A-silicone impression materials. Both Fix and Silfix Adhesives need to be applied in a thin layer ensuring economy due to less material wastage. This thin layer produces an extremely strong adhesion. Fix solvent is available for easy removal of the remaining adhesive. Reader response number 56

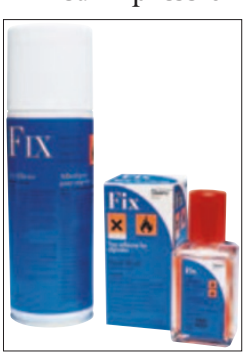

\section{New bonding system}

Kemdent have used the latest nano-technology bonding system in the new Diamond Bond light-curing, self-etching adhesive. Diamond Bond enables dentists to combine the advantages of Diamond Carve GIC (Glass ionomer Cement) with aesthetic benefits offered by the new Diamond Finish composite technology in a single procedure. This saves valuable chair time and produces superior restoration outcomes.

When you order Diamond Bond 2 x 4ml pack @ £56.60 you will receive a free Diamond Finish Packable refill $2 \times 4 \mathrm{~g}$ worth $£ 41.10$. Offer ends 31 July 06. For more information visit website www. kemdent.co.uk Reader response number 57

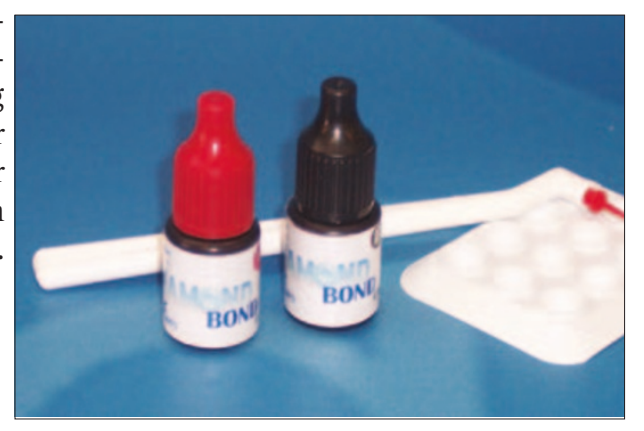




\section{Power with control}

The PrepStart air abrasion system from Evident combines power and control facilitates to provide a range of procedures. It ensures greater precision and conservation when cutting

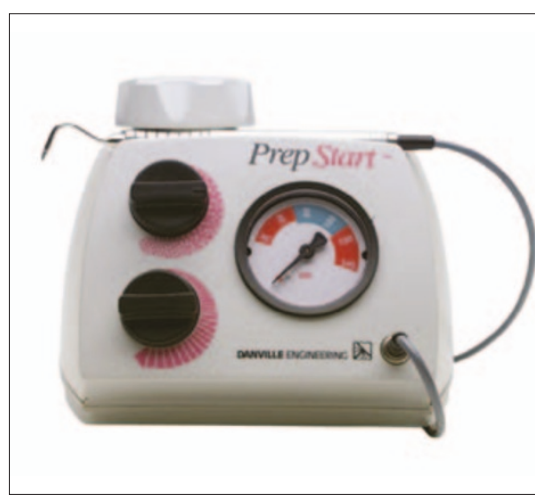
and etching than is found with conventional handpieces and is the ideal tool for identifying and treating fissure lesions.

The PrepStart handpiece does not come into contact with teeth at any time, virtually eliminating noise and vibration. Patient discomfort is minimised, reducing the need for local anaesthetics, lessening chairside time and allowing for multiple treatments to be performed in a single visit.

Finally, the neat and compact unit means it fits easily into all surgeries.

Reader response number 58

\section{Fast strong temporaries}

Integrity, the temporary crown and bridge material from Dentsply is available in a dispensing system that virtually eliminates cross-contamination and provides fast, strong temporaries together with aesthetics. It has good consistency for easy handling and clean-up and bonds to itself for easy repair. It polishes to a natural lustre and with less than a $5 \mathrm{C}$ temperature change in the mouth during setting, it will not damage the pulp.

The product offers patient comfort while minimal shrinkage and adjustment can save you time. Due to a high flexural and transverse strength, Integrity has a high degree of resistance to marginal fracture. It is available in five shades, including bleach white.

Reader response number 59
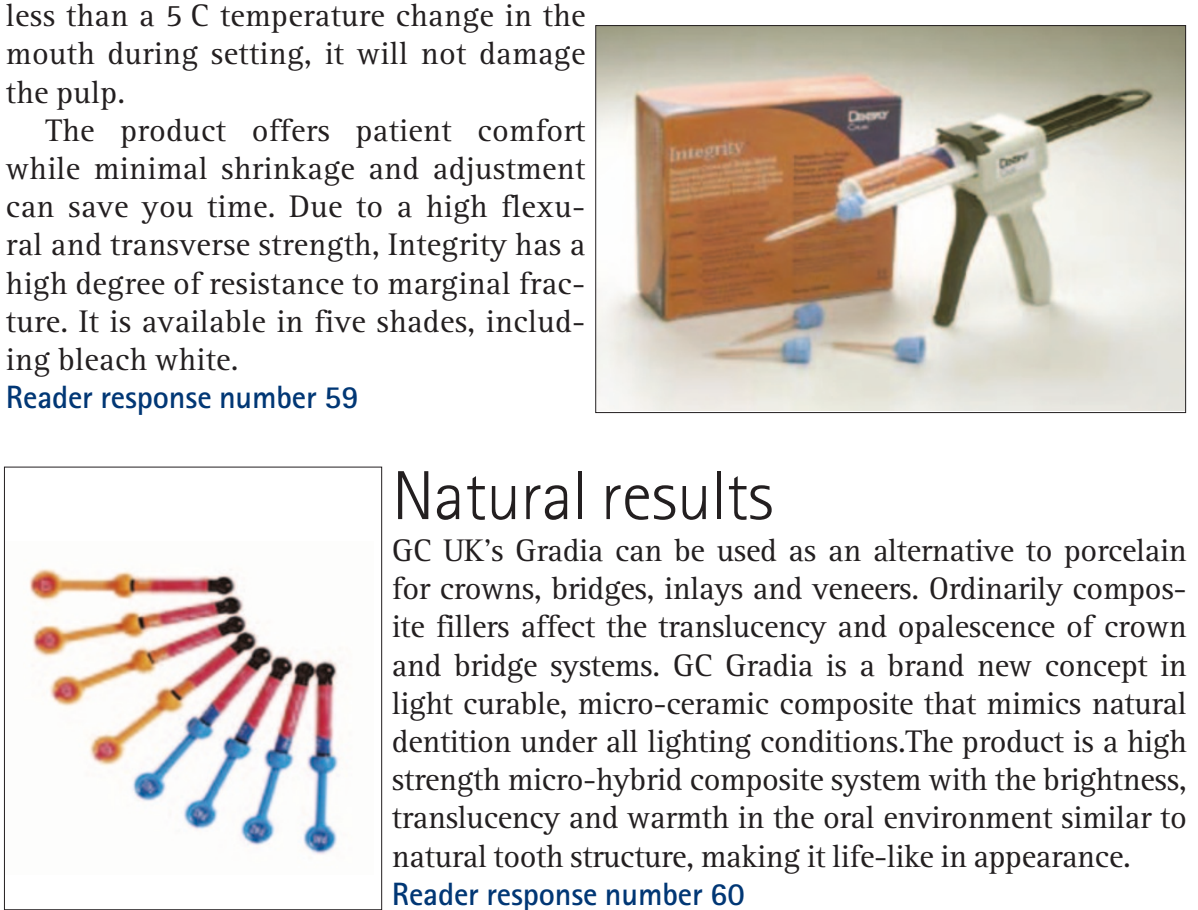

Natural results

GC UK's Gradia can be used as an alternative to porcelain for crowns, bridges, inlays and veneers. Ordinarily composite fillers affect the translucency and opalescence of crown and bridge systems. GC Gradia is a brand new concept in light curable, micro-ceramic composite that mimics natural dentition under all lighting conditions. The product is a high strength micro-hybrid composite system with the brightness, translucency and warmth in the oral environment similar to natural tooth structure, making it life-like in appearance. Reader response number 60

\section{New Amalgam Well launched}

Astek Innovations have recently launched the Pegasus Amalgam Well, an addition to its range of Amalgam Carriers. The new Pegasus Well is machined from a solid block of stainless steel, carefully bottom weighted to prevent spillages, and comes with a handy anti slip o-ring for even more security and is fully autoclavable.

Its range of plastic Amalgam Carriers are manufactured from high grade, autoclavable material and have a smooth action for long term ease of use. Controlled ejection of amalgam is provided with the option of three interchangeable nozzles (straight, $45^{\circ}$ and $90^{\circ}$ ) which are also available separately. Currently with every four Pegasus Amalgam Carriers purchased at $£ 6.50$ each a free Pegasus Amalgam Well is available, making a saving of £12.95. Pegasus Amalgam Carriers are available in straight, $45^{\circ}$ and $90^{\circ}$ angle versions.

Reader response number 61

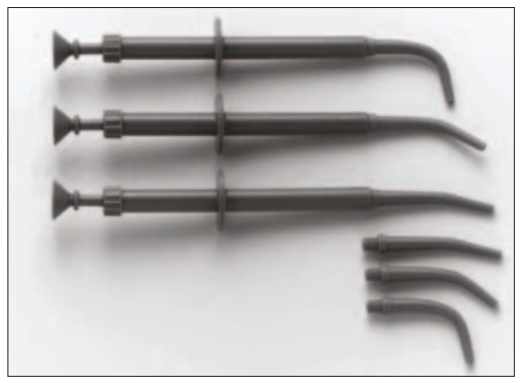

